\title{
Efectos de la inclusión de contenidos de desarrollo personal en un programa de educación parental para familias en riesgo psicosocial
}

\author{
Margarita Peña, María L. Máiquez* y María J. Rodrigo
}

Facultad de Psicología. Universidad de La Laguna (España)

\begin{abstract}
Resumen: Este estudio analiza los resultados del Programa de Apoyo Personal y Familiar para familias en riesgo psicosocial, teniendo en cuenta los efectos inmediatos obtenidos sobre la agencia parental y las prácticas educativas de los participantes. Los resultados muestran que la inclusión de contenidos de desarrollo personal junto con los de habilidades educativas permiten mantener, e incluso mejorar, los resultados obtenidos en estas variables en anteriores ensayos en los que sólo se incluyeron contenidos de habilidades educativas. Asimismo, como novedad frente a anteriores evaluaciones, se pudo comprobar que dichos resultados no se ven modulados por el nivel de riesgo psicosocial de la familia. Por último, junto a este enfoque centrado en variables se adopta un enfoque centrado en las personas para analizar los distintos patrones de cambio encontrados entre los participantes en las dimensiones anteriormente estudiadas y las variables que permiten caracterizar cada tipología de cambio. En la discusión se destaca la pertinencia de incluir dichos contenidos en la educación parental con familias en riesgo psicosocial.

Palabras clave: Desarrollo personal; habilidades educativas; intervención grupal; familias en riesgo psicosocial.
\end{abstract}

Title: Effects of including personal development content on parenting program for families at psychosocial risk.

Abstract: This study analyses the results of the Personal and Familiar Support Program targeted to families at psychosocial risk, with regard to its immediate effects obtained on the parental agency and the educational practices of the participants. The results showed that the incorporation of personal development contents together with those of educational skills allowed for improving the results obtained in previous trials in which only contents of educational skills were included. Likewise, as a novelty with respect to previous evaluations, it was obtained that the above results were not modulated by the level of family psychosocial risk. Finally, in addition to the variable-centered approach, a person-centered approach was adopted to analyze the different pattern of change found among the participants in the dimensions previously studied, and the variables that characterize every typology of change. The discussion emphasizes the relevance of including personal development contents in the parent education in families at psychosocial risk.

Key words: Personal development; educational skills; group intervention; families at psychosocial risk.

\section{Introducción}

En la actualidad cada vez son más frecuentes las acciones destinadas a que madres y padres reciban apoyos educativos para el desempeño del rol parental. Este hecho no ha de extrañarnos si tenemos en cuenta que el desarrollo de dicho rol se hace especialmente difícil en la sociedad actual, no sólo por la complejidad que encierra la tarea, sino por la gran variedad de formas familiares existentes, que implican a su vez una enorme diversificación en el modo de llevar a cabo la realización de la tarea de ser madres y padres (Martínez González, 2009; Rodrigo, Máiquez, Martín y Byrne, 2008; Triana y Rodrigo, 2010).

Asimismo, tampoco podemos olvidar que todas las familias requieren de aliados y apoyos sociales para llevar a cabo la tarea de crianza y socialización de sus hijos e hijas (Barudy y Dantagnan, 2007; 2010; Gracia, 2011; Palusci, 2011; Rodrigo et al., 2008). Sin embargo, estas necesidades de apoyo, no son iguales para todas las familias, sino que éstas dependen tanto de las características de las madres y los padres como de las necesidades educativas de los hijos e hijas y del contexto psicosocial de la familia, caracterizado por tener más o menos factores de protección y de riesgo (Bronfenbrenner y Evans, 2000; Cicchetti y Valentino, 2006; Rodrigo, Máiquez y Martín, 2010).

Precisamente, el Consejo de Europa, a través de la Recomendación Rec (2006) 19, resalta la importancia de las acciones psicoeducativas, en forma de programas de educación parental, como recurso de apoyo preventivo y de promoción

* Dirección para correspondencia [Correspondence address]: María Luisa Máiquez. Campus de Guajara. Facultad de Psicología. Universidad de La Laguna. Tenerife. E-mail: mmaicha@ull.es para el fomento de la parentalidad positiva. En especial, la educación parental cobra relevancia para aquellas familias que necesitan de mayor apoyo en su tarea como son las familias en riesgo psicosocial. En este caso, la Recomendación destaca la conveniencia de organizar programas y acciones formativas con metodologías grupales que permitan a las madres y a los padres compartir y contrastar sus experiencias parentales y apoyarse mutuamente en la adquisición de estrategias que fomenten el desarrollo de un ejercicio positivo de la parentalidad.

El programa de Apoyo Personal y Familiar ya inició su andadura en 1997 con este enfoque de prevención y promoción de las capacidades parentales, especialmente aquellas ligadas a las habilidades educativas. En el año 2001 con ocasión de su implementación en los servicios sociales municipales se incluyeron también contenidos de desarrollo personal. Esta decisión se basó en la observación, constatada por los profesionales, de que una buena parte de las carencias presentadas en las madres y padres de familias en situación de riesgo tiene que ver con las competencias relativas a su desarrollo como adultos, convirtiéndose en un importante obstáculo que condiciona el desarrollo de una parentalidad positiva. Estos nuevos contenidos están dirigidos a potenciar las capacidades personales y relacionales para fomentar los factores de resiliencia de estas familias. Sin pretender cambios radicales en estas familias, el objetivo es ir cambiando aspectos concretos de su vida cotidiana; la forma en la que se perciben, lo qué sienten, cómo se relacionan con el entorno o cómo plantean su futuro. Con ello, se persigue potenciar las capacidades personales y contribuir así al desarrollo de las madres y los padres (Rodrigo et al., 2008; Rodrigo et al., 2010).

A medida que se ha ido adquiriendo una visión más completa y elaborada sobre las características y necesidades 
las familias en riesgo psicosocial también han ido evolucionando las formas de atención e intervención (Hidalgo, Menéndez, Sánchez, Lorence y Jiménez, 2009; Máiquez, Rodrigo, Capote y Vermaes, 2000; Martín, Máiquez, Rodrigo, Correa y Rodríguez, 2004; Olds, 2012; Rodrigo, Correa, Máiquez, Martín y Rodríguez, 2006; Rodrigo, Martín, Máiquez y Rodríguez, 2007). Sin embargo, pese a la gran variedad existente de programas de apoyo a la familia, no hemos encontrado en la literatura programas que trabajen, específicamente, el desarrollo personal de las madres y los padres en situación de riesgo psicosocial.

Sabemos que la condición de riesgo psicosocial implica no sólo dificultades y obstáculos para lograr el bienestar psicológico y social, sino que también afecta al desarrollo personal y comunitario (Barudy y Dantagnan, 2010; BuenoAbad, 2005: Gracia, 2011; Garbarino y Eckenrode, 1999; Martín-Baró, 1998; Moreno, 2001). Así, las madres y los padres que viven en estas condiciones presentan una gran dificultad para integrar las experiencias emocionales vividas a lo largo de su biografía personal, plena de eventos vitales negativos, dificultando así el logro de una identidad personal adulta. También llevan a cabo un análisis reducido y simplista de las situaciones vitales y de las experiencias personales. Asimismo, presentan escasas habilidades de toma de decisión y de resolución de conflictos, lo que se asocia a una baja percepción de las propias capacidades (autoeficacia). Por último, presentan un proyecto de vida desdibujado o ausente, lo que se traduce en el establecimiento de metas personales no elegidas sino impuestas socialmente, una baja implicación y compromiso vital, elección de medios inadecuados para alcanzar metas y un proyecto de vida poco realizable o incluso ausente.

Con el Programa de Apoyo Personal y Familiar tratamos de fortalecer y promover no sólo habilidades parentales sino todas aquellas competencias, habilidades y recursos personales que promueven el desarrollo personal de estas madres y padres en riesgo psicosocial. Así, tenemos por un lado, contenidos de habilidades educativas, desarrollados en cinco módulos: actividades de la vida cotidiana; las madres y los padres ante los problemas del desarrollo; comunicación y resolución de conflictos en la familia; situaciones que cambian la vida familiar y las respuestas de las madres y los padres ante situaciones difíciles; y por otro, hemos elaborado unos contenidos de desarrollo personal a lo largo de cuatro nuevos módulos: identificar la vida personal; analizar las situaciones personales; resolver situaciones personales; elaborar mi proyecto de vida.

Estos nuevos módulos permiten promover las competencias que son básicas para normalizar y regularizar el proceso de desarrollo personal y social. En concreto, mediante la realización de estos módulos se promueven una serie de dimensiones de cambio: de una identidad personal fragmentada a la consecución de una identidad lograda; de un análisis simple de las situaciones vitales a una capacidad para evaluar de manera compleja las situaciones y afrontar lo adverso; de escasas habilidades en resolución de conflictos a mejorar las habilidades de comunicación y solución de conflictos, reforzando las fuentes de apoyo; de un proyecto de vida desdibujado o ausente a la elaboración de un proyecto de vida realista. En suma, se trata de crear personas competentes socialmente, que tengan conciencia de su identidad y utilidad, que puedan tomar decisiones, establecer metas y creer en un futuro mejor, satisfacer sus necesidades básicas de afecto, relación y respeto en todos los ámbitos de su vida e incrementar el apoyo social de la familia.

El programa de Apoyo Personal y Familiar en su versión original con contenidos en habilidades parentales ya había sido evaluado anteriormente (Martín et al., 2004; Rodrigo, Martín, Máiquez y Rodríguez, 2005). En dicha evaluación se observó que los efectos son muy notables en la agencia parental, observándose una mayor autoeficacia, locus de control interno, percepción más realista de la dificultad de su rol y mayor acuerdo con la pareja al terminar el programa. También es positivo que se hayan podido modificar, aunque más modestamente, las pautas educativas ya que después del programa los padres y madres eran más inductivos y menos permisivo-negligentes.

Con este estudio queremos comprobar si se mantienen los mismos resultados del programa al incorporar algunos módulos de desarrollo personal. Confiamos que se mantengan los resultados positivos en la agencia parental y en las pautas educativas, a pesar de destinar menos tiempo a los contenidos de habilidades parentales ya que el programa que se evalúa contiene tres módulos de habilidades parentales y dos de desarrollo personal. Se introducen también dos novedades: a) comprobar hasta qué punto los resultados se ven influidos por los niveles de riesgo psicosocial de las familias y b) analizar la variabilidad en los tipos de cambio observados tras el programa en las variables de agencia parental y las prácticas educativas, Asimismo, se exploran las características individuales y grupales que nos permiten caracterizar cada tipología de cambio.

\section{Método}

\section{Participantes}

En este estudio han participado 140 madres, de las que 72 formaron el grupo de intervención y 68 el grupo control. El grupo de intervención estuvo formado por aquellas madres referidas por los Servicios Sociales Municipales de la isla de La Palma, que realizaban el Programa de Apoyo Personal y Familiar en el curso 2001-2002. Aunque el programa era para padres y madres, no participó en el programa ningún padre, ya que suele ser habitual la presencia minoritaria de los padres en este tipo de programas.

Se formaron 12 grupos de madres, uno por cada servicio social de los municipios que estaban participando en el programa. La media de participantes por grupo fue de 5.8 (DT = 3.6). El porcentaje de abandono del programa fue del $32 \%$. De un total de 115 madres que comenzaron el programa, 72 madres lo siguieron y formaron el grupo de inter- 
vención. Los motivos de abandono informados por las madres fueron característicos de las intervenciones de larga duración: cambio de residencia, situaciones inesperadas (accidente, enfermedad), nuevo trabajo y embarazo. Las participantes que abandonaron y aquellas que completaron el programa no diferían en las variables sociodemográficas ni en las medidas pre-test.

En relación al grupo control, éste estaba compuesto por las madres que iban a intervenir en la siguiente edición del programa en las mismas zonas de la isla. Por razones éticas no fue posible escoger al azar madres para el grupo control. No obstante, no existían diferencias significativas en ninguna de las variables sociodemográficas planteadas entre el grupo de intervención y el grupo control. El grupo de intervención se caracterizó por ser madres con una media de 38.69 (DT $=13.70)$ años y 2.56 (DT =1.56) hijos, con estado civil casado en un $45.7 \%$, y en un $62.3 \%$ biparentales. El 88.6 residían en zonas rurales, con un nivel educativo tanto propio $(52.9 \%)$ como de sus parejas $(52.4 \%)$ sin estudios o estudios primarios. Se trataba de familias que en un $56.7 \%$ no perciben ayudas económicas, están en situación de desempleo $(70.6 \%)$ al igual que sus parejas (64.5\%).

\section{Instrumentos}

Perfil sociodemográfico de las familias. Este instrumento consiste en una ficha que debe cumplimentar el propio participante facilitando algunos datos sociodemográficos como son: sexo, edad, número de hijos/as, estado civil, si tienen hijos/as en el sistema de protección, si tienen hijos/as con necesidades educativas especiales, nivel educativo propio $\mathrm{y}$ de la pareja, situación económica y situación laboral, tanto propia como de la pareja.

Protocolos de evaluación del riesgo psicosocial (Rodríguez, Camacho, Rodrigo, Martín y Máiquez, 2006). Con una versión para familias biparentales y otra para familias monoparentales y respondidos por el Técnico de Servicios Sociales, analizan 42 indicadores con respuestas dicotómicas si/no, en las siguientes áreas; factores sociodemográficos (8 ítems; p.e., nivel educativo parental, número de hijos/as); apoyo social familiar (2 ítems; p.e., apoyo de la familia extensa); organización familiar y doméstica ( 7 ítems; p.e., vivienda inadecuada, falta de organización en el hogar, falta de control sanitario); historia de maltrato y características personales de los cuidadores (7 ítems; p.e., historia de abuso del padre/madre; abuso de drogas por parte de los padres/madres; madre con depresión); calidad de las relaciones dentro de la familia (7 ítems; p.e., relaciones conflictivas padre/madre-hijos/hijas, relación violenta dentro de la pareja); prácticas educativas inadecuadas (5 ítems; p.e., negligencia parental, normas rígidas e incoherentes); problemas de adaptación en los hijos/as (6 ítems; p.e., problemas de conducta, problemas emocionales).

El instrumento ofrece tres coeficientes discriminantes para cada indicador de acuerdo al nivel de riesgo (bajo, medio, alto). Un simple cálculo consistente en la suma por cada nivel de riesgo de los coeficientes de cada indicador aplicable a la familia más una constante, permite estimar el nivel de riesgo de la familia que corresponde a la suma más alta alcanzada en los tres niveles.

En la investigación que nos ocupa, la distribución de los niveles de riesgo fue la siguiente: un $40.3 \%$ se clasificó como familias de riesgo bajo (con escasos indicadores de riesgo), el $16.7 \%$ como familias de riesgo medio (con indicadores relativos a desventaja psicosocial), y el $43.1 \%$ como familias de riesgo alto (con indicadores de desventaja psicosocial, violencia y maltrato en la familia).

Cuestionario sobre la agencia parental de los padres y las madres (Máiquez, Capote, Rodrigo y Martín, 1999). Este cuestionario aporta información sobre la auto-percepción de las madres y los padres con respecto a su propio rol parental a través de cuatro dimensiones comunes en los dos tiempos de medida; Control Interno: con 4 ítems (alpha de Cronbach = .82) que valoran el grado en que una madre o un padre tiene la percepción de ser un agente activo y transformador de la realidad familiar, a través de cuestiones como "Creo que puedo controlar los comportamientos de mis hijos e hijas"; Autoeficacia: con 5 ítems (alpha de Cronbach $=.80$ ) que valoran el grado en que una madre o un padre tiene la percepción de tener la capacidad de resolver diversos problemas familiares, por medio de cuestiones como "El hecho de ser padre o madre me ha supuesto ser más responsable"; Acuerdo en la pareja: Con 2 ítems (alpha de Cronbach $=.75$ ) que valoran el grado en que una madre o un padre tiene la percepción de que existe acuerdo y apoyo entre él/ella y su pareja, a través de cuestiones como " Mi pareja y yo estamos de acuerdo en la forma de educar a nuestros hijos e hijas"; Percepción de la dificultad del rol parental: con 4 ítems (alpha de Cronbach $=.82$ ) que valoran el grado en que una madre o un padre tiene la percepción de que la tarea parental es una tarea difícil, a través de cuestiones como "Creo que después de tantos años me va a ser difícil cambiar mi forma de educar" y una quinta dimensión que varía en función del momento de medida; al inicio se evalúa la Motivación para el cambio; con 4 ítems (alpha de Cronbach $=.79$ ) que valoran el grado en que una madre o un padre tiene expectativas positivas con respecto a su posibilidad de cambio, a través de cuestiones como "Creo que necesito ayuda para mejorar la forma de educar a mis hijos e hijas" y al finalizar el programa se evalúa la Transferencia a la vida cotidiana; con 4 ítems (alpha de Cronbach $=.79$ ) que valoran el grado en que una madre o un padre tiene la percepción de que es capaz de trasladar lo aprendido a su propia vida cotidiana, a través de cuestiones como, "Ahora, cuando ocurre un problema en mi familia, enseguida me doy cuenta de cómo tengo que reaccionar".

La escala está compuesta por 19 ítems valorados en escalas tipo likert de siete puntos, donde 0 corresponde a nada y 6 corresponde a mucho, en la que mayores puntuaciones se corresponden con un mayor nivel de las dimensiones analizadas. Este instrumento requiere de aproximadamente unos 10 minutos para su cumplimentación y se aplica de forma 
auto-administrada.

Cuestionario situacional de prácticas educativas de riesgo (adaptado de Ceballos y Rodrigo,1992). Esta prueba aporta información sobre los métodos de crianza de los hijos e hijas a través de seis situaciones en la que aparecen algunas conductas problemáticas de externalización (p.e., "Una tarde su hijo/a se encuentra solo/a en casa y está muy aburrido. Aunque tiene prohibido usar el radio casete de su hermano, lo coge y se lo rompe"; de internalización (p.e., "Su hijo/a llega a casa muy triste y a punto de llorar. El motivo es que unos compañeros de clase se han burlado de él/ella y no le han dejado jugar con ellos"), y de transgresión social (p.e., "Usted se encuentra al profesor de su hijo/a. Éste le dice que no presta atención en clase, interrumpe con frecuencia y molesta a los compañeros y compañeras"). Cuenta con dos formas paralelas, una de ellas se aplica al inicio del programa y otra al finalizar el mismo. Explora tres prácticas educativas; Práctica Permisivo-Negligente: con 10 ítems en la versión inicial y 8 ítems en la versión final, (alpha de Cronbach $=.76$ ), p.e., “ Le digo al profesor que le deje actuar así porque es su forma de ser"; Práctica Inductiva: con 5 ítems en la versión inicial y 9 ítems en la versión final (alpha de Cronbach $=.79$ ) p.e., "Le recomiendo a mi hijo que hable con su amigo y entre ambos resuelvan sus diferencias" y Práctica Coercitiva: con 5 ítems en la versión inicial y 4 ítems en la versión final, (alpha de Cronbach $=.80$ ) p.e., "Le doy una nalgada a tiempo para que no lo vuelva a hacer". Las prácticas son exploradas a través de cuatro ítems valorados en una escala de cinco puntos $(0=$ Nunca, $1=$ Pocas veces, 2 = Algunas veces, $3=$ Casi siempre, $4=$ Siempre) en cada una de las situaciones problemáticas anteriormente mencionadas, por tanto hablamos de un total de 24 ítems, donde una mayor puntuación indica una mayor utilización de la práctica educativa que representa. Este instrumento requiere de aproximadamente unos 15 minutos para su cumplimentación y se aplica de forma autoadministrada.

\section{Procedimiento}

Los cuestionarios iniciales se administraron durante las dos sesiones iniciales. Los cuestionarios finales se completaron al finalizar el programa. La persona responsable de cada grupo conocía los instrumentos de evaluación y estaba capacitada para resolver cualquier duda que pudiera surgir, gracias a la formación previa llevada a cabo por los responsables del programa.

\section{Diseño de evaluación y estrategias de análisis}

Utilizamos un diseño cuasi experimental, consistente en un grupo de intervención con prueba previa y posterior y un grupo control en espera de tratamiento y con características similares al grupo de intervención. Para dar respuesta a los objetivos de investigación planteados hemos realizado comparaciones pre-postest, comparaciones intergrupo sólo en el post-test y se han realizado análisis de conglomerados para explorar la variabilidad de cambios pre-post en el grupo de intervención.

Así, en primer lugar con el fin de analizar los cambios producidos en el grupo de intervención tras su participación en el Programa se exploraron los cambios en la agencia parental de las familias tales como el control interno, la autoeficacia, el acuerdo en la pareja y la percepción de la dificultad de su rol parental, así como cambios en las prácticas educativas permisivo-negligentes, inductivas y coercitivas. Este análisis comenzó con una exploración inicial a través de análisis multivariados de varianza (MANOVA) para cada una de las dos dimensiones analizadas, tomando como variables de medida la propia dimensión y el tiempo de medida. En todos los contrastes realizados se empleó la $F$ ofrecida por Lambda de Wilks para la interpretación de la significatividad del modelo. En este caso se exploró el tamaño del efecto a partir del estadístico $\eta^{2}$ parcial (Cohen, 1988). Para las comparaciones pre-postest de los factores de las diversas dimensiones planteadas se emplearon análisis de medidas repetidas en dos tiempos de medida inicial y final, empleando $F$ para la interpretación de la significatividad del contraste. En este caso se exploró el tamaño del efecto a partir del estadístico $R^{2}$, (Cohen, 1988). Asimismo, se realizaron análisis de medidas repetidas de las diferencias pre-postest incluyendo variables sociodemográficas como la edad, el número de hijos/as, existencia en la familia de hijos/as con necesidades especiales, existencia de hijos/as en centros de protección, la zona (rural o urbana), el estado civil, la tipología familiar (monoparental o biparental), el nivel de estudios, el nivel de estudios de la pareja, la situación económica (percibir o no ayudas económicas), situación laboral propia y de la pareja (desempleo- trabajador) la motivación para el cambio (baja o alta) y el nivel de riesgo, así como una variable referida al modo de implementación, que es el tamaño del grupo. Se realizaron análisis post hoc Scheffe, para aquellas variables de diferentes niveles (edad, nivel de estudios, estado civil y $\mathrm{n}^{\circ}$ de hijos/as). Por último, se realizaron análisis multivariados entre el grupo de intervención y de control sólo en el post-test, procediéndose a la misma exploración de resultados que se hizo con el contraste pre-test y post-test del grupo de intervención.

En segundo lugar, para nuestro análisis centrado en las personas y el estudio de los tipos de cambio, en las dimensiones de agencia parental y prácticas educativas, que se observan tras su participación en el programa, se realizó en primer lugar, para determinar la variabilidad, un análisis de conglomerados jerárquico; y en segundo lugar, se realizó un análisis de conglomerados confirmatorio, empleando el procedimiento de conglomerados con $\mathrm{k}$ medias. Una vez determinados los conglomerados se realizó un análisis de las características de los participantes pertenecientes a cada conglomerado a partir de análisis univariantes de varianza (ANOVA) en el caso de que la variable dependiente fuera cuantitativa. En cambio, cuando los análisis implicaban la comparación de grupos en función de variables dicotómicas se empleó el estadístico $\chi^{2}$ (Chi-Cuadrado) para la obtención 
del nivel de significación del contraste, realizando comparaciones de frecuencias y determinando la magnitud de la acumulación de las mismas en las distintas categorías a través del análisis de los residuos tipificados corregidos. En este caso se exploró el tamaño del efecto a partir del estadístico $v$ de Cramer (Agresti, 1996).

\section{Resultados}

\section{Cambios producidos en el grupo de intervención}

En relación a la dimensión agencia parental, en los MANOVA se obtuvo una interacción significativa entre ésta y la variable tiempo de medida $\left(F_{(3,67)}=3.576 p \leq .05, \mathrm{y}^{2}=\right.$ .14). De igual forma se obtuvo una interacción significativa entre la variable prácticas educativas y la variable tiempo de medida $\left(F_{(2,68)}=3.544, p \leq .05, \mathrm{y}^{2}=.14\right)$. Ello indica que los cambios se dieron más en algunas variables de agencia parental que en otras. Los resultados del análisis de medidas repetidas para cada una de las variables de cambio planteadas en agencia parental y prácticas educativas pueden verse en la Tabla 1 donde se presentan las medias y desviaciones típicas pre-postest para el grupo de intervención, en cada una de las variables, así como su contraste.

Como puede observarse, estos resultados pusieron de manifiesto la existencia de diferencias pre-postest en las dos variables exploradas. En relación a las variables de agencia parental se observó una única diferencia estadísticamente significativa en el aumento de la autoeficacia, mostrando una relevancia clínica alta. En relación a las prácticas educativas se observaron diferencias significativas en dos de las prácticas analizadas, con una disminución de las prácticas permisivo-negligentes y las prácticas coercitivas, mostrando una relevancia clínica alta.

Tabla 1. Contraste de medias pre-postest en el grupo de intervención.

\begin{tabular}{|c|c|c|c|c|c|}
\hline & $\begin{array}{l}\text { Pretest } \\
M(D T)\end{array}$ & $\begin{array}{l}\text { Postest } \\
M(D T)\end{array}$ & $F$ & $g l$ & $\begin{array}{c}\text { Tamaño del efecto } \\
\left(R^{2} \text { parcial }\right)\end{array}$ \\
\hline \multicolumn{6}{|l|}{ Agencia Parental } \\
\hline Control Interno & $4.44(1.22)$ & $4.28(1.06)$ & 1.076 & $1 / 69$ & .98 \\
\hline Autoeficacia & $4.93(.61)$ & $5.20(.63)$ & $7.208^{* *}$ & $1 / 69$ & .91 \\
\hline Acuerdo Pareja & $3.81(2.07)$ & $3.24(2.50)$ & 3.738 & $1 / 69$ & .94 \\
\hline $\begin{array}{l}\text { Percepción dificultad } \\
\text { Del rol }\end{array}$ & $3.08(1.31)$ & $3.02(1.00)$ & .145 & $1 / 69$ & .99 \\
\hline \multicolumn{6}{|l|}{ Prácticas Educativas } \\
\hline Permisivo-negligente & $.88(.53)$ & $.57(.45)$ & $19.480^{* * *}$ & $1 / 69$ & .79 \\
\hline Coercitiva & $1.63(.81)$ & $1.09(.73)$ & $19.752^{* * *}$ & $1 / 69$ & .78 \\
\hline Inductiva & $2.71(.64)$ & $2.81(.58)$ & .950 & $1 / 69$ & .98 \\
\hline
\end{tabular}

${ }^{* * *} p \leq .001 * * p \leq .01 * p \leq .05$

Diferencias postest entre el grupo de intervención y el grupo control

En relación a la dimensión agencia parental, se obtuvo una interacción significativa entre la variable agencia parental y la variable grupo de pertenencia $\left(F_{(4,117)}=4.142, p \leq .01\right.$, $\mathrm{y}^{2}=.13$ ), indicando que existían diferencias en la variable agencia parental en función del grupo de pertenencia. Así, se observaron dos diferencias significativas en esta variable entre el grupo de intervención y el grupo control, en concreto en la variable autoeficacia y en la variable acuerdo en la pareja (ver Tabla 2).

Tabla 2. Contraste de medias en el post-test entre el grupo de intervención y el grupo control en Agencia Parental y Prácticas Educativas.

\begin{tabular}{|c|c|c|c|c|c|}
\hline 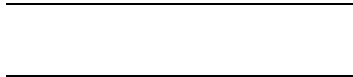 & $\begin{array}{c}\text { Grupo de intervención } \\
M(D T)\end{array}$ & $\begin{array}{c}\text { Grupo Control } \\
M(D T)\end{array}$ & $F$ & $g l$ & Tamaño del efecto ( $R^{2}$ parcial $)$ \\
\hline \multicolumn{6}{|l|}{ Agencia Parental } \\
\hline Control Interno & $4.28(1.06)$ & $4.11(1.28)$ & .773 & $1 / 133$ & .01 \\
\hline Autoeficacia & $5.20(.63)$ & $4.85(.71)$ & $4.432 *$ & $1 / 125$ & .03 \\
\hline Acuerdo Pareja & $3.24(2.50)$ & $4.15(1.9)$ & $9.488 * *$ & $1 / 126$ & .07 \\
\hline Percepción dificultad del rol & $3.02(1.00)$ & $3.19(.98)$ & .051 & $1 / 130$ & .05 \\
\hline \multicolumn{6}{|l|}{ Prácticas Educativas } \\
\hline Permisivo-negligente & $.57(.45)$ & $1.38(1.50)$ & $19.003 * * *$ & $1 / 133$ & .13 \\
\hline Coercitiva & $1.09(.73)$ & $1.68(1.4)$ & $9.249 * *$ & $1 / 133$ & .07 \\
\hline Inductiva & $2.81(.58)$ & $2.71(.64)$ & 3.273 & $1 / 133$ & .02 \\
\hline
\end{tabular}

$* * * p \leq .001 * * p \leq .01 * p \leq .05$

Los análisis univariantes recogidos en la Tabla 2 indican que las variables que contribuyeron específicamente a explicar las diferencias existentes entre el grupo de intervención y el grupo control fueron la autoeficacia $\left(F_{(1,125)}=4.432 ; p \leq\right.$
.05) y el acuerdo en la pareja $\left(F_{(1,126)}=9.448 ; p \leq .01\right)$. Así, el nivel de autoeficacia resultó ser más alto en el grupo de intervención que en el grupo control mientras que el acuerdo en la pareja fue más alto en el grupo control. 
En relación a la dimensión prácticas educativas, se obtuvo una interacción significativa entre la variable prácticas educativas y la variable grupo de pertenencia $\left(F_{(3,131)}=6.302\right.$ $\left.p \leq .001, \mathrm{y}^{2}=.13\right)$, indicando que existían diferencias en la variable prácticas educativas en función del grupo de pertenencia. Así, se observaron dos diferencias significativas en esta variable entre el grupo de intervención y el grupo control, en concreto, en las prácticas permisivo-negligentes y en las práctica coercitivas (ver Tabla 4), donde se presentan los análisis univariados (ANOVAS) realizados con cada una de las variables analizadas de las prácticas educativas. Estos resultados indican que las variables que han contribuido a explicar las diferencias existentes entre el grupo de intervención y el grupo control son las prácticas permisivonegligentes y las prácticas coercitivas. El grupo de intervención presentó menos prácticas permisivas y menos coercitivas en relación al grupo control

\section{Variables que modulan las diferencias pre-postest en el grupo de intervención}

Respecto a la agencia parental, las ganancias en control interno al finalizar el programa estuvieron moduladas por la variable tener menores en un centro de acogida $\left(F_{(1,64)}=\right.$ $5.088, p \leq .05)$. En concreto, aquellas madres que tenían hijos/as en el sistema de protección mostraron mayores ganancias en control interno, una vez finalizado el programa, que aquellas madres que no los tenían.

Las ganancias en autoeficacia al finalizar el programa estuvieron moduladas por la variable edad de las madres $\left(F_{(3,60)}\right.$ $=4.601, p \leq .05)$ fueron las madres de más de 40 años las que mostraron mayores ganancias en autoeficacia en comparación con aquellas madres menores de 25 años. Asimismo, aquellas madres que sí tenían hijos/as en el sistema de protección mostraron mayores ganancias en autoeficacia, una vez finalizado el programa, que las madres que no los tenían $\left(F_{(1,64)}=2.068, p \leq .05\right)$. Por último, las madres cuyas parejas no tenían estudios o estudios primarios alcanzaron mayores niveles de autoeficacia en comparación con aquellas madres con Graduado o FP $\left(F_{(2,39)}=1.542, p \leq .05\right)$.

Las ganancias en acuerdo en la pareja en temas educativos, al finalizar el programa, estuvieron moduladas por la edad de las madres $\left(\mathrm{F}_{(3,66)}=3.081, p \leq .05\right)$, ya que fueron las madres entre 40-50 años las que mostraron mayores ganancias en acuerdo en la pareja que aquellas madres mayores de 50 años.

En relación a la percepción de dificultad en el rol parental, los efectos fueron modulados por el tamaño del grupo, $\left(F_{(1,68)}\right.$ $=5.700, p \leq .05)$ y por la motivación para el cambio $\left(F_{(1,68)}\right.$ $=6.523, p \leq .05)$. En concreto, fueron aquellas madres que acudían a grupos pequeños, esto es, menos de 5 personas las que obtuvieron mayores incrementos en la percepción de la dificultad del rol que aquellas que acudían a grupos medios, esto es entre 5 y 10 personas. Por su parte, mostraron una mayor dificultad en el rol las madres que presentaban baja motivación para el cambio en comparación con aquellas que presentaban alta motivación para el cambio.

Respecto a las prácticas educativas, los efectos en las prácticas permisivo-negligentes, al finalizar el programa estuvieron modulados por tener hijos/as con necesidades educativas especiales $\left(F_{(1,69)}=4.985, p \leq .05\right)$, por el nivel de estudios de la pareja $(F=3.967, p \leq .05)$ y por la situación económica $\left(F_{(2,39)}=7.405, p \leq .01\right)$. Concretamente, fueron las madres que tenían hijos/as con necesidades educativas especiales las que más disminuyeron las prácticas permisivonegligentes frente a las que no los tenían. Así como, las madres cuyas parejas no tenían estudios o estudios primarios en comparación con aquellas madres con Graduado o FP y aquellas madres que si percibían ayudas económicas frente a las que no las percibían, fueron las que más disminuyeron dichas prácticas.

Las ganancias en prácticas inductivas, estuvieron moduladas por la variable edad de la madre, $\left(F_{(1,54)}=5.635, p \leq .001\right)$, por el número de hijos/as $\left(F_{(4,65)}=8.901, p \leq .001\right)$ y por tener menores en centros de protección $\left(F_{(1,64)}=17.968\right.$, $p$ $\leq .000)$. En concreto, las madres que tenían entre 30 y 40 años realizaban un mayor número de prácticas inductivas en comparación con aquellas madres que tenían entre 40 y 50 años y aquellas que tenían más de 50 años. Las madres que tenían 3 ó 4 hijos/as, en comparación con aquellas madres que tenían menos o más de 6 hijos eran las que realizaban un mayor número de prácticas inductivas. Al igual que las madres que tenían hijos/as en centros de protección.

\section{Tipologías de cambio en las familias}

Se analizó visualmente el dendograma, obtenido como resultado del procedimiento de conglomerados jerárquico exploratorio, para la toma de decisiones relativa al número de conglomerados homogéneos. Así, se observó que favorecía la selección de tres o cuatro conglomerados. Teniendo en cuenta los criterios de interpretabilidad de los grupos, la adecuación de su tamaño y la capacidad para diferenciarse entre sí, se tomó la decisión de contar con cuatro conglomerados. En la Figura 1 se reflejan los valores promedio obtenidos en cada uno de los conglomerados en las distintas dimensiones de cambio estandarizadas consideradas. 


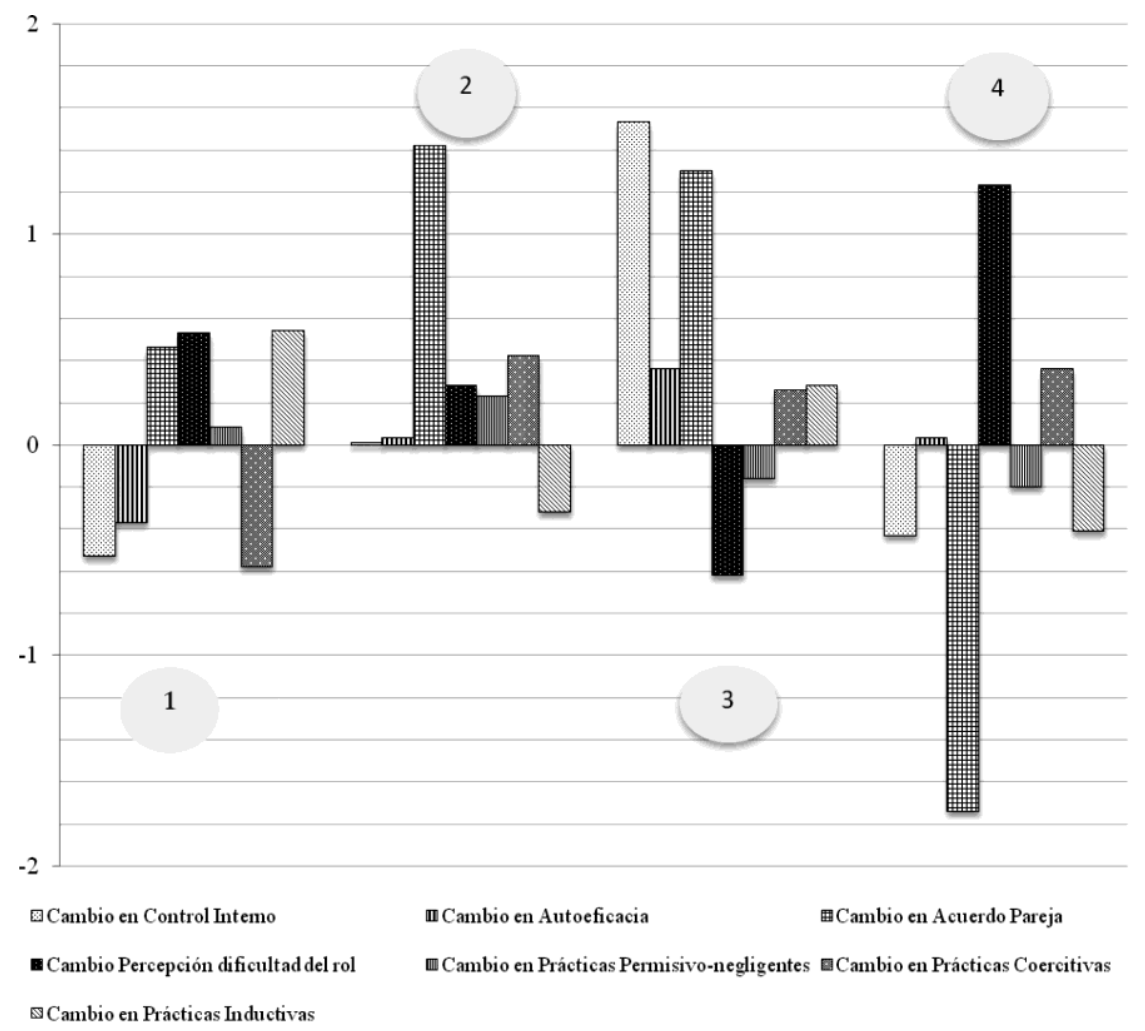

Figura 1. Centros de los conglomerados finales de la solución obtenida en el procedimiento de conglomerados k medias con 4 clusters $(n=61)$.

Los distintos conglomerados difirieron en todas las variables de cambio analizadas, excepto en las prácticas permisivo-negligentes e inductivas. Este resultado se puede ver en la Tabla 3, donde se presentan los contrastes realizados y las pruebas post hoc para cada uno de los conglomerados.

Tabla 3. Centros de los conglomerados finales y contrastes univariados de varianza entre los distintos conglomerados en función de las dimensiones de cambio $(n=61)$.

\begin{tabular}{|c|c|c|c|c|c|c|c|}
\hline \multirow[b]{2}{*}{ Dimensiones } & \multicolumn{4}{|c|}{ Conglomerados } & \multirow[b]{2}{*}{$F(3,57)$} & \multirow[b]{2}{*}{$R^{2}$} & \multirow[b]{2}{*}{ Post hoc } \\
\hline & $\begin{array}{c}1 \\
(n=19)\end{array}$ & $\begin{array}{c}2 \\
(n=14)\end{array}$ & $\begin{array}{c}3 \\
(n=15)\end{array}$ & $\begin{array}{c}4 \\
(n=13)\end{array}$ & & & \\
\hline Cambio Control Interno & -.53 & .01 & 1.53 & -.43 & $18.444 * * *$ & .49 & $\begin{array}{c}1-3 * * * \\
2-3 * * * \\
3-4 * * *\end{array}$ \\
\hline Cambio Autoeficacia & -.37 & .03 & .36 & .03 & $3.115^{*}$ & .03 & $1-3^{*}$ \\
\hline Cambio Acuerdo Pareja & .46 & 1.42 & 1.30 & $-1,74$ & $24.086^{* * *}$ & .56 & $\begin{array}{l}1-4 * * * \\
2-4 * * * \\
3-4 * * *\end{array}$ \\
\hline Cambio Percepción dificultad del rol parental & .53 & .28 & -.62 & 1.23 & $18.816^{* * *}$ & .49 & $\begin{array}{l}1-2^{* * *}, 1-4^{*} \\
2-3^{* * *} \\
3-4^{* * *}\end{array}$ \\
\hline Cambio Prácticas Permisivo-negligentes & .08 & 23 & -.16 & -.20 & 1.55 & .08 & \\
\hline Cambio Prácticas Coercitivas & -.58 & .42 & .26 & .36 & $4.366^{*}$ & .08 & $1-2^{*}$ \\
\hline Cambio Prácticas Inductivas & .54 & -.32 & .28 & -.41 & $4.265^{*}$ & .09 & 1-4* \\
\hline
\end{tabular}

A continuación, describimos las dimensiones más relevantes de cada conglomerado, considerando los valores promedio obtenidos por cada grupo y las diferencias establecidas a nivel estadísticamente significativo. La denominación de los clusters, se realizó teniendo en cuenta el criterio de amplitud de los cambios (total o parcial). Así, un cambio total indica que el conglomerado mostró diferencias a nivel es- tadísticamente significativo en las dos dimensiones de cambio (agencia parental y prácticas educativas). Un cambio parcial indica que el conglomerado mostró diferencias a nivel estadísticamente significativo únicamente en una de las dimensiones de cambio. Asimismo, se tuvo en cuenta si los cambios eran positivos, negativos o mixtos en cada conglomerado: 
Conglomerado 1: Cambio total mixto. Este conglomerado estaba compuesto por participantes que disminuyeron en control interno y en autoeficacia. Así como, disminuyeron las prácticas coercitivas y aumentaron las prácticas inductivas tras su participación en el programa.

- Conglomerado 2: Cambio total mixto. Este grupo de participantes se caracterizó por presentar un aumento en el acuerdo en la pareja. Así como por presentar un aumento de prácticas educativas coercitivas tras su participación en el programa.

- Conglomerado 3: Cambio parcial positivo. Los participantes pertenecientes a este conglomerado mostraron un aumento del control interno, así como aumentaron su sentimiento de autoeficacia y disminuyeron su percepción en la dificultad del rol tras su participación en el programa.

- Conglomerado 4: Cambio total mixto. Este grupo de participantes mostró una disminución en acuerdo en la pareja y un aumento en su percepción de la dificultad del rol tras su participación en el programa. Así como una disminución de las prácticas educativas inductivas.

\section{Caracterización de las tipologías de cambio de las familias}

Los resultados de los diferentes análisis nos muestran que no existieron diferencias estadísticamente significativas en relación a las variables sociodemográficas examinadas. De modo que las madres que conformaron los conglomerados no se distinguieron de forma significativa por ninguna de estas variables. Sin embargo para la variable motivación para el cambio de las familias los resultados de la prueba Chicuadrado $\left(\chi^{2}\right)$ pusieron de manifiesto la existencia de diferencias significativas en función de la motivación para el cambio en los distintos conglomerados considerados $\left(\chi^{2}=9.170, p=\right.$ $.02)$, con una relevancia clínica media $(V=.39)$. Los residuos tipificados corregidos mostraron cómo las madres con alta motivación para el cambio se acumularon en el tercer conglomerado (cambio parcial positivo). Asimismo, los resultados del análisis para la variable tamaño del grupo nos muestran que no existieron diferencias estadísticamente significativas.

\section{Discusión y Conclusiones}

La evaluación del programa, realizada con la implementación de tres módulos de habilidades parentales y dos de desarrollo personal, nos muestra que con la inclusión de contenidos de desarrollo personal, los resultados del programa parecen ir en la línea de lo encontrado anteriormente en la evaluación del Programa de Apoyo Personal y Familiar (Martín et al., 2004; Rodrigo et al., 2005). El grado de seguimiento del programa fue un poco más bajo $(70 \%)$ pero hay que tener en cuenta que la mayor parte de los abandonos tuvieron causas técnicas y no lo fueron tanto por razones de desinterés o pérdida motivacional. Ello no es extraño pues este programa cuida mucho todos aquellos factores que pueden facilitar la presencia de las madres como servicio de guardería, transporte, llamadas telefónicas, etc.

En relación a la dimensión de agencia parental, las madres tras realizar el programa aumentaron significativamente su percepción de autoeficacia. Esto es, las madres una vez finalizado el programa perciben tener más capacidad para educar a sus hijos e hijas y tienen mayor confianza en su rol. Este resultado se mantiene al compararlo con las madres del grupo control. Sabemos que el desarrollo de la tarea parental se encuentra directamente vinculado a las competencias personales de las madres y padres, reflejando el modo en que los progenitores perciben y viven su rol parental (Azar, 1998; Máiquez et al., 2000). En este sentido, este resultado es importante en cuanto que es reconocido que un mayor nivel de autoeficacia lleva a las personas a persistir ante las tareas difíciles lo que puede garantizar la continuidad en el esfuerzo (Bandura, 1982). Sin embargo, el grado de acuerdo con la pareja fue menor en el grupo de intervención, resultado éste que hemos obtenido en otras ocasiones ya que el programa pone en evidencia la precariedad del apoyo educativo del padre lo cual disminuye la percepción de acuerdo parental en temas educativos (Martín et al., 2004).

En relación a las prácticas educativas el programa fue efectivo en la disminución de las prácticas educativas de riesgo. Las madres informan que tras el programa hacen un menor uso de prácticas coercitivas y prácticas permisivonegligentes. Este resultado se mantiene al compararlo con el grupo control. Esta disminución de las prácticas permisivonegligentes y coercitivas presenta especial interés en las familias en situación de riesgo psicosocial, ya que estas se caracterizan por sostener dichas prácticas y proyectarlas en el uso de una disciplina incoherente (Rodrigo et al., 2008).

Otro resultado interesante, es que la interacción entre el nivel de riesgo y las puntuaciones pretest y postest no fue estadísticamente significativa, por tanto las ganancias no variaron según el nivel de riesgo de las familias participantes. En un estudio previo de una versión piloto del programa con una duración de cuatro meses y con contenidos de habilidades parentales, fueron las madres y padres de riesgo bajo quienes obtuvieron más beneficios tras realizar el mismo (Máiquez et al., 2000), por tanto se confirma la eficacia de nuestro programa para las familias en riesgo psicosocial independientemente del nivel de riesgo presentado.

Por otro lado, las madres que han visto incrementada en mayor medida su agencia parental presentaron un perfil caracterizado por una alta motivación inicial, con edades comprendidas entre los 40 y 50 años, con menores en el sistema de protección y cuyas parejas estaban desempleadas. Asimismo las madres que disminuyeron las prácticas educativas de riesgo respondieron a aquellas que presentaban un perfil de vulnerabilidad psicosocial, caracterizadas por ser receptores de ayudas económicas debido a la situación de pobreza 
en la que se encuentran (Martín et al., 2004; Moreno, 2001), con bajo nivel educativo y desempleo (Trigo, 1992), y ser familias monoparentales con un subsistema conyugal poco estable (Nieto, 1996), todas ellas características que se corresponden con el perfil de los principales destinatarios del programa.

En suma, se ha demostrado la capacidad del Programa de Apoyo Personal y Familiar de fortalecer las competencias parentales, incidiendo tanto sobre la agencia parental como sobre las prácticas educativas en madres con distintos niveles de riesgo psicosocial. Estos efectos beneficiosos se ven potenciados en el caso de familias que presentan un perfil sociodemográfico de mayor vulnerabilidad social y personal.

La imagen global de los efectos del Programa de Apoyo Personal y Familiar desarrollada nos ha permitido extraer conclusiones interesantes con respecto al cambio en diversos componentes de las competencias parentales. Sin embargo, este tipo de análisis no permite apresar la heterogeneidad que podría existir en los procesos de cambio de las madres que participan en el mismo. En este sentido, hemos realizado un esfuerzo por examinar esta posible heterogeneidad asumiendo una perspectiva de análisis centrada en el propio individuo, es decir, en los procesos de cambio de las madres (Magnusson y Stattin, 2006).

Los conglomerados encontrados presentan procesos de cambio muy variados. Así, se obtuvo que el $75.41 \%$ de las madres mostraron cambios mixtos, tanto positivos como negativos y el $24.59 \%$ de las madres cambios parciales positivos. Asimismo es interesante constatar que abundan los perfiles en los que las madres mejoran en agencia parental pero no en las prácticas educativas y viceversa, lo cual nos indica que son procesos de cambio independientes. El aprendizaje de una adecuada parentalidad es complejo, ya que se compone de un proceso objetivo y un proceso subjetivo que deben darse ambos para generar un adecuado desarrollo del rol parental de un modo integral (Stark, Gruber, Renal y Mandl, 1998). En este sentido, el proceso objetivo de aprendizaje hace referencia al cambio en las prácticas educativas de los progenitores mientras que el proceso subjetivo hace referencia a su percepción del ejercicio de la parentalidad, es decir, la agencia parental. Lo que hemos observado es que en la mayoría de las madres $(75.41 \%)$ existe todavía una disociación entre los dos procesos, indicando que el

\section{Referencias}

Agresti, A. (1996). Introduction to categorical data analysis. Nueva York: John Wiley

Azar, S. (1998). Parenting and child maltreatment. En I. E. Sigel y K. A. Renninger (Eds.), Child Psychology in practice (Volume 4.) (pp. 361-388). Handbook of Child Psychology. (5 ${ }^{\text {th }}$ edition) (W. Damon, Ed.). New York: John Wiley y Sons.

Bandura, A. (1982). Self-efficacy mechanism in agency. American Psychologist, $37,122-147$.

Barudy, J. y Dantagnan M. (2007). Los buenos tratos en la infancia: parentalidad, apego y resiliencia. Gedisa.

Barudy, J., y Dantagnan, M. (2010). Los desafíos invisibles de ser madre o padre: Manual de evaluación de las competencias y resiliencia parental. Gedisa aprendizaje está en fase transicional hacia la consecución de la integración de ambos. Lo que indica que a corto plazo el programa no ha podido consolidar todo el aprendizaje complejo, ya que éste se encuentra aún en transición. Quizás una medida de cambio a largo plazo sea capaz de evaluar la consecución final de dicho aprendizaje complejo.

Por otro lado, no encontramos características asociadas a las familias ni al tamaño de los grupos que pudiera explicar la adscripción de las madres a los diferentes perfiles. Una excepción es el perfil de cambio parcial positivo con beneficios en agencia parental, donde fueron las madres con alta motivación las que tenían una mayor representación en este conglomerado. Parece pues que esta motivación para el cambio sirve de activador y facilitador de ese aprendizaje complejo que sólo hemos observado de forma consolidada en ese $24.59 \%$ de las madres.

En definitiva, creemos que la inclusión de contenidos de desarrollo personal en los programas de padres dirigidos a familias en riesgo psicosocial, puede frenar las influencias negativas que las situaciones estresantes que viven estas familias ejercen sobre el desarrollo de los hijos. Sin embargo, los resultados de esta primera evaluación señalan la necesidad de incluir el resto de los contenidos de desarrollo personal para lograr mayores efectos sobre la agencia personal. Asimismo, otro reto es elaborar instrumentos para realizar una evaluación a medio y largo plazo que recoja los cambios producidos en aquellos aspectos entrenados directamente en dichos contenidos como son la capacidad de afrontamiento y resolución de conflictos, las habilidades comunicativas, la búsqueda de apoyos sociales y la orientación hacia el futuro. De este modo, en próximos ensayos del programa podremos evaluar plenamente las aportaciones de estos contenidos de desarrollo personal.

Agradecimientos.- Queremos expresar nuestro agradecimiento a los técnicos de los Equipos de riesgo de los Servicios Sociales Municipales de la Isla de La Palma, y a las madres que participaron en el programa. La implementación y evaluación del Programa de Apoyo Personal y Familiar, se realizó gracias al acuerdo alcanzado entre el Cabildo de La Palma, la Fundación Canaria Radio ECCA y la Universidad de La Laguna. La evaluación se realizó con el apoyo del proyecto concedido por el Ministerio de Ciencia e Innovación (TRA2009_0145).

Bronfenbrenner, U., y Evans, G. W. (2000). Developmental science in the $21^{\text {st }}$ century: emerging theoretical models, research designs, and empirical findings. Social Development, 9, 115-125. doi: 10.1111/14679507.00114

Bueno-Abad, J. R. (2005). El proceso de ayuda en la intervención psicosocial. Madrid: Popular .

Ceballos, E.M. y Rodrigo, M.J. (1992). A situational questionnaire of child-rearing practices and goals. Comuncación presentada en la $\mathrm{V}^{\text {th }}$ European Conference on Developmental Psychology (6-9 Septiembre). Sevilla, España.

Cicchetti, D. y Valentino, K. (2006). An ecological-transactional perspective on child maltreatment: failure of the average expectable environment and its influence on child development. En Cohen, D. y Cicchetti, D. 
(Ed.), Developmental psychopathology, vol. 3: Risk, disorder, and adaptation (2 $2^{\text {nd }}$ ed.) (pp.129-201). Hoboken, NJ, US: John Wiley y Sons.

Cohen, J. (1988). Statistical power analysis for the behavioural sciences (2nd ed.) Hillsdale, NJ: Erlbaum.

Hidalgo, M.V., Menéndez, S., Sánchez, J., Lorence, B. y Jiménez, L. (2009). La intervención con familias en situación de riesgo psicosocial. Aportaciones desde un enfoque psicoeducativo. Apuntes de Psicología, 27, (2-3), 413-426.

Garbarino, J. y Eckenrode, J. (1999). Por qué las familias abusan de sus hijos. Barcelona: Granica, S.A

Gracia, E. (2011). Apoyo social e intervención social y comunitaria En I Fernández, J. F. Morales y F. Molero (Coords.), Psicología de la intervención comunitaria (pp. 129-171). Bilbao: Desclée de Brower.

Magnusson, D. y Stattin, H. (2006). The person in context: A holisticinteractionistic approach. En R. M. Lerner y W. Damon y R. M. Lerner (Eds.), Handbook of child psychology: Vol. 1. Theoretical models of human development ( $6^{\mathrm{a}}$ ed.) (pp. 400-464). Nueva Jersey: John Wiley.

Máiquez, M. L., Capote, C., Rodrigo, M. J. y Martín, J. C. (1999). Cuestionario sobre la agencia personal de los padres y de las madres. Departamento de Psicología Evolutiva y de la Educación. Universidad de La Laguna. No publicado.

Máiquez, M. L., Rodrigo, M. J., Capote, C. y Vermaes, I. (2000). Aprender en la vida cotidiana: un programa experiencial para padres. Madrid: Visor.

Martín-Baró, I. (1988), Psicología de la liberación. Madrid: Ed. Trotta

Martín, J. C., Máiquez, M. L., Rodrigo, M. J., Correa, A. D. y Rodríguez, G. (2004). Evaluación del programa "Apoyo personal y familiar" para madres y padres en situación de riesgo psicosocial. Infancia y Aprendizaje, 27, 437-445.

Martínez González, R. A. (2009). Programa-Guía para el Desarrollo de Competencias Emocionales, Educativas y Parentales. Madrid: Ministerio de Sanidad y Política Social.

Moreno, P. (2001), Concepto de marginación social. En P. Moreno (Ed.), Psicología de la marginación social; concepto, ámbitos y actuaciones (pp. 67-101). Málaga: Ed. Aliibe

Nieto, L. M. (1996): Familias pobres y multiasistidas. En M. Millán (Dir.) Psicología de la familia. Un enfoque evolutivo y sistémico (pp. 201-227). Valencia: Promolibro.
Olds D. L. (2012). Improving the Life Chances of Vulnerable Children and Families with Prenatal and Infancy Support of Parents: The NurseFamily Partnership. Psychosocial Intervention, 21, 129-143. doi: $10.5093 /$ in $2012 \mathrm{a} 14$

Palusci, V. J. (2011). Risk factors and services for child maltreatment among infants and young children. Children and Youth Services Review, 33, 1374 1382.

Recomendación Rec (2006) 19 Politicas de apoyo al ejercicio positivo de la parentalidad. http://www.coe.int/t/dg3/youthfamily

Rodríguez, G. Camacho, J., Rodrigo, M. J., Martín, J. C., y Máiquez, M. L. (2006). Evaluación del riesgo psicosocial en familias usuarias de servicios sociales municipales. Psicothema, 18 (2), 200-206.

Rodrigo, M. J., Correa, A. D., Máiquez, M. L., Martín, J. C. y Rodríguez, G. (2006). Family preservation services in Canary Islands: Predictors of the efficacy of a Parenting program for families at-risk of social exclusion. European Psychologist, 11 (1), 57- 70. doi: 10.1027/1016-9040.11.1.57

Rodrigo, M. J., Máiquez, M. L y Martín, J. C. (2010). La educación parental como recurso psicoeducativo para el apoyo de la prentalidad positiva. Madrid: Publicaciones de la Federación Española de Municipios y Provincias.

Rodrigo, M. J., Máiquez, M. L., Martín, J. C. y Byrne, S. (2008). Preservación familiar. Un enfoque positivo para la intervención con familias. Madrid: Pirámide

Rodrigo, M. J., Martín, J. C., Máiquez, M. L., y Rodríguez, G. (2005). El asesoramiento a familias con riesgo psicosocial. En C. Monereo y J. I. Pozo (Eds.), La práctica asesora a examen (pp. 40-70). Barcelona: Graó.

Rodrigo, M. J., Martín, J. C., Máiquez, M. L. y Rodríguez, G. (2007). Informal and formal supports and maternal child-rearing practices in at-risk and non at-risk psychosocial contexts. Children and Youth Services Review, 29, 329-347

Stark, R., Gruber, H., Renkl, A. y Mandl, H. (1998). Instructional effects in complex learning: do objetive and subjetive learning outcomes converge? Learning and Instruction, 8(2), 117-130.

Triana, B. y Rodrigo, M. J. (2010). Modelos y estrategias de intervención ante la diversidad familiar. En E. Arranz y A. Oliva (Eds.), Desarrollo psicológico en las nuevas estructuras familiares (pp. 121-142). Editorial Pirámide.

Trigo, J. (1992): Familia e infancia en riesgo psicosocial. Apuntes de Psicología, $34,51-58$.

(Articulo recibido: 10-05-2012; revisado: 03-03-2013; aceptado: 03-03-2013) 